\title{
Data prediction for cases of incorrect data in multi-node electrocardiogram monitoring
}

\author{
Sugondo Hadiyoso ${ }^{1,2}$, Heru Nugroho ${ }^{1,2}$, Tati Latifah Erawati Rajab ${ }^{1}$, Kridanto Surendro ${ }^{1}$ \\ ${ }^{1}$ School of Electrical and Information Engineering, Bandung Institute of Technology, Bandung, Indonesia \\ ${ }^{2}$ School of Applied Science, Telkom University, Bandung, Indonesia
}

\begin{tabular}{l} 
Article Info \\
\hline Article history: \\
Received Apr 29, 2021 \\
Revised Sep 11, 2021 \\
Accepted Oct 10, 2021 \\
\hline
\end{tabular}

Keywords:

Expectation-maximization

Incorrect data

Predict

Regression imputation

Root mean square error

\begin{abstract}
The development of a mesh topology in multi-node electrocardiogram (ECG) monitoring based on the ZigBee protocol still has limitations. When more than one active ECG node sends a data stream, there will be incorrect data or damage due to a failure of synchronization. The incorrect data will affect signal interpretation. Therefore, a mechanism is needed to correct or predict the damaged data. In this study, the method of expectationmaximization (EM) and regression imputation (RI) was proposed to overcome these problems. Real data from previous studies are the main modalities used in this study. The ECG signal data that has been predicted is then compared with the actual ECG data stored in the main controller memory. Root mean square error (RMSE) is calculated to measure system performance. The simulation was performed on 13 ECG waves, each of them has 1000 samples. The simulation results show that the EM method has a lower predictive error value than the RI method. The average RMSE for the EM and RI methods is 4.77 and 6.63, respectively. The proposed method is expected to be used in the case of multi-node ECG monitoring, especially in the ZigBee application to minimize errors.
\end{abstract}

This is an open access article under the $\underline{C C B Y-S A}$ license.

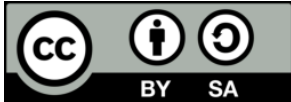

\section{Corresponding Author:}

Sugondo Hadiyoso

School of Electrical and Information Engineering, Bandung Institute of Technology

School of Applied Science, Telkom University

Bandung, Indonesia

Email: sugondo@telkomuniversity.ac.id

\section{INTRODUCTION}

Nowadays, there are advanced progress in applying computing technologies [1]-[8], that have significant progress in artificial intelligence. The development of wireless communication media on the internet of things application is always followed by the development of protocols to support multiple or multiuser access. Multiuser monitoring or control applications have been applied in one of them in the health area. This application allows for centralized, fast, easy, remote, and multiuser health monitoring. Health parameters that get serious attention are the heart of this refers to the risks posed if not maintained optimally. Observation of heart conditions can be done by studying the electrical activity of the heart through an electrocardiogram (ECG) [9]-[11]. Previous research by Hadiyoso and Aulia [12], has succeeded in designing and implementing an ECG monitoring system for several ZigBee-based user nodes. But in its application, there are crucial problems, namely damage or loss of data if more than one active node is sending data streams [13]. This problem is likely to occur because of the failure of synchronization between the user/end node and the coordinator.

Estimating missing data is a significant advancement that occurs during the data cleaning stage. Numerous studies have demonstrated that improper data management results in inaccurate analysis [14]. 
Missing data, as indicated by the absence of data items for a subject, can obscure some potentially significant information. In practice, missing data has emerged as a significant determinant of data quality. Thus, the imputation of the missing value is needed [15]. Missing data is a common weakness in the classification problem, and it can cause the prediction system's output to be ineffective [16], [17]. Ignoring missing data has an effect on the analysis's results [18], [19], the outcomes of learning, as well as the outcomes of predictions on the collaborative prediction problem [20]. In quantitative studies, missing data leads to biased parameter estimates [21]-[24]. In the predictive model, the selection of methods for handling incorrect data missing can affect model performance [22], [25]. Missing data are common in medical research, and if not handled properly, they can result in a loss of statistical power and potentially biased results [26]-[28]. The standard data collection problems may involve noiseless data. In addition to the presence of noisy data, organizations face challenges with the presence of missing data. Missing data will affect extensive data collection, so investigating different filtering techniques for large data environments will be extraordinary [29]. This proposed study will not discuss or observe for the cause of the problem, rather than how to improve or predict the incorrect data with a technique which is commonly used in the case of missing data. This is the urgency of the research proposed to provide a reliable telemonitoring system with the smallest possible error rate to avoid misinterpretation.

Several methods have been applied to predict missing data in various applications. In general, missing value imputation techniques fall into two categories: Statistical and machine learning-based techniques [30]. Expectation-maximization (EM), linear regression (LR), least squares (LS), and mean/mode are the four statistical techniques that are most frequently used [31]. The use of EM in the imputation of missing data has several advantages including missing data does not need to be ignored so that it can increase information for the accuracy of diagnosis [32] and can handle many patterns of missing data [33]. Imputation using linear regression results in a small standard deviation [34], although regression imputation is better than average imputation but results in biased parameter estimates [35]. Expert methods such as support vector machines (SVM) and artificial neural networks (ANN) used in data prediction were also reported in the study [36], [37]. However, this method has high computational costs and is complex to be implemented in computers with low memory resources.

The literature study above provides enough knowledge as a basis for the proposed study. Research on predictions of missing data using a mathematical approach provides enough evidence to be applied to solve problems with ZigBee-based multiuser monitoring implementation. In this study, we applied a method to overcome the incorrect data, they are EM and regression linear imputation. This study aims to predict the incorrect data and determine the best method between the two proposed methods. Performance analysis is done by calculating the root mean square error (RMSE) between the reconstruction data and the actual data stored on the microcontroller memory. As a reminder, the rest of the paper is organized as follows: section 2, containing an explanation of the data collection and the proposed method for handling missing data. In section 3, we explain the results and discussion of the simulations that have been carried out. In section 4 we present the conclusions and implications of this study.

\section{MATERIAL AND METHOD}

\subsection{Data collection}

ECG data is sourced from previous studies which are real data from the streaming of each user node. This multipoint ECG network uses a mesh topology where a coordinator is used to receive data from other nodes. The coordinator was connected to the personal computer (PC), then the PC displays the ECG chart according to the active node. ECG data from each node is also stored in memory by the microcontroller to be used for comparing the performance or comparison in calculating RMSE. Figure 1 is an example of a graph of an ECG signal that has errors in nodes $\mathrm{C}$ and $\mathrm{D}$. At node $\mathrm{C}$, the graph that is marked shows the data value of 0 , this causes the ECG waveform to be distorted. Meanwhile, at node D, the chart marked shows that the data has risen significantly like a spike (reaching a value of 1024 in decimal). Some of the other point samples were also omitted manually randomly to test the robustness of the proposed method. This also causes the ECG waveform to be distorted. These conditions become the main problem when there are errors in data transmission. This phenomenon is then considered to be a problem of incorrect data.

\subsection{Method for handling missing data \\ 2.2.1. Regression imputation (RI)}

In the regression model, the observed values are used to calculate missing values. The assumed value is then used to fill in the blanks where the missing value was previously present. Like mean imputation, this method has the advantage of selecting more information from which to determine the appropriate imputation value [38]. A technique known as regression imputation is used to replace missing values with predictive values that are estimated primarily based on the data that is available. Missing variables have a 
significant impact on which regression imputation technique is used [39]. Imputation regression begins by calculating the average vector estimation and covariance matrix of data based on sub-matrices that contain data without missing values [40]. Following that, the observed data is used to calculate linear regression for missing variables. The values obtained from linear regression are then used to replace missing data. However, the variance and covariance of data are underestimated using this method [41].

Consider the case of univariate non-response, where Y1; ...; YK-1 are completely observed and YK are observed for the first $\mathrm{r}$ observations but missing for the last $\mathrm{n}-\mathrm{r}$ observations. Regression imputation is a method of calculating the regression of a set of variables YK on Y1; ... YK-1 fills in the missing values using the $\mathrm{r}$ complete cases using the regression predictions [40].

$$
\hat{y}_{i K}=\tilde{\beta}_{K 0 \cdot 12 \ldots K-1}+\sum_{j=1}^{K-1} \tilde{\beta}_{K j \cdot 12 \ldots K-1} y_{i j}
$$

Where, $\widetilde{\beta}_{K 0 \cdot 12 \ldots K-1}$ is the intercept and $\widetilde{\beta}_{K j \cdot 12 \ldots K-1}$ is the coefficient of $\mathrm{Yj}$ in the regression of $\mathrm{Y}_{\mathrm{K}}$ on $\mathrm{Y}_{1} ; \ldots$; $\mathrm{Y}_{\mathrm{K}-1}$ based on the $r$ complete cases.

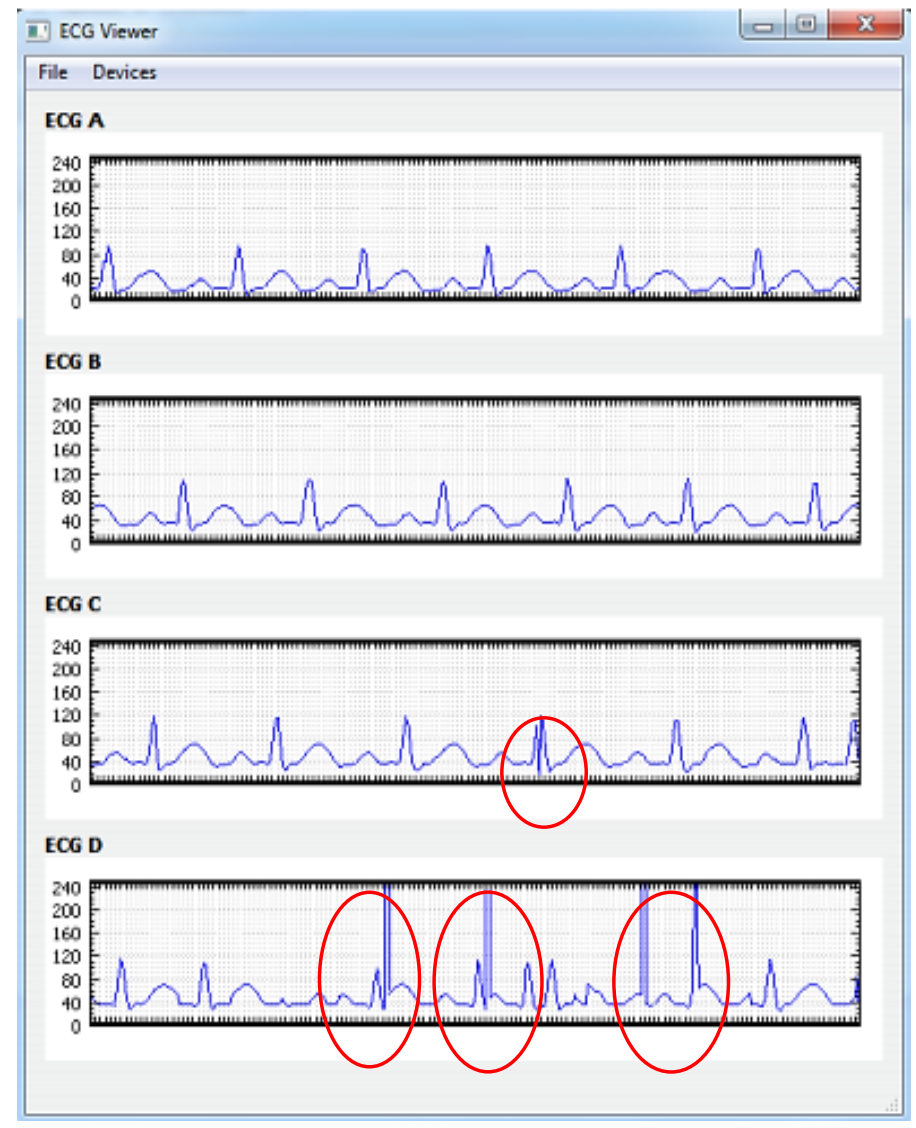

Figure 1. Examples of ECG waves that have errors [5]

\subsubsection{Expectation-maximization (EM) imputation}

The maximum possibility is a mathematical procedure for determining one or more statistical model parameters for observed data by maximizing the observed probability distribution. The EM algorithm is a recursive algorithm for determining the maximum probability estimation parameter in the presence of incomplete data. EM Imputation is a two-step iterative procedure that utilizes the maximum probability method. We begin by inferring the unobserved values from the expected value (E-step). In the second step, the expected value is constructed using the maximum of the initial values (M-step). After that, this EM cycle is repeated until the imputation values satisfy the specified convergence criteria. The EM imputation method generates an unbiased estimate of the standard parameter [41]-[44]. 
Specifically, allow to be the most recent estimate of the parameter. It is the E step of the EM procedure that determines the expected complete-data loglikelihood if were to assume: Specifically, let $\theta^{(t)}$ be the current estimate of the parameter $\theta$. The expected complete-data loglikelihood is found in the E step of EM if $\theta$ were $\theta^{(t)}$ :

$$
Q\left(\theta \mid \theta^{(t)}\right)=\int \ell(\theta \mid y) f\left(Y_{m i s} \mid Y_{o b s}, \theta=\theta^{(t)}\right) d Y_{m i s}
$$

where, $Y_{m i s}$ is the missing data and $Y_{o b s}$ is observed data. The M step in EM establishes $\theta^{(t+1)}$ maximization of the expected complete-data loglikelihood (observed for all $\theta$ :

$$
Q\left(\theta^{(t+1)} \mid \theta^{(t)}\right) \geq Q\left(\theta \mid \theta^{(t)}\right) \text { for all } \theta
$$

\subsection{Root mean square error (RMSE)}

The root mean square error (RMSE) is one parameter that can be used to determine the accuracy of continuous variables. In terms of error magnitude, the RMSE is measured. RMSE is defined mathematically as the square root of the average square difference between the predicted model and the actual value. In other words, RMSE quantifies the degree of congruence between observed and predicted data. The RMSE equation as (4):

$$
R M S E=\sqrt{\frac{1}{n} \sum_{j=1}^{n}\left(y_{j}-\hat{y}_{j}\right)^{2}}
$$

where, $y_{j}$ is actual data; $\hat{y}_{j}$ is prediction data; and $n$ is number of data.

\section{RESULTS AND DISCUSSION}

Typically, missing data follows discernible patterns. Investigating this pattern is critical for identifying instances and variables that contribute to the missing data [43], [45]. Figure 2 shows the pattern of missing data on ECG waves which were observed in this study. It is shown that the missing data pattern for the ECG dataset is general. To make it easier to understand the missing values patterns that were used for the analysis variables, the patterns chart has been greatly enlarged to increase its interpretability. Every pattern (row) corresponds to a bunch of cases with a similar pattern of incomplete and complete data. In other words, wherever the missing values are located, the patterns or groups of cases are displayed in support (i.e., on each variable). For instance, pattern 1 denotes cases with no missing values, whereas pattern 4 denotes cases with missing values on the ECG3. It is expected that all missing cells and non-missing cells in the chart will be contiguous if the data is monotone. The pattern of missing values from the dataset is non-monotone and there are several values that ought to be imputed. As a result, the monotonicity of this data is not demonstrated, and the monotone method of imputation is not justified in this situation. Some methods for dealing with missing data are applicable to any pattern of missing data, whereas other methods are only applicable to a specific pattern of missing data.

This study simulates the sending of ECG data streams from 4 nodes where each is connected to an ECG amplifier to record the subject's ECG waveform. At the same time, data is transmitted to the personal computer and stored in memory by the controller unit. The application software will display a signal graph and save the data stream in .txt format to match the data which is stored in memory. In this study, 13 subjects were involved in recording ECG data through these nodes. ECG data in the decimal format of 1000 samples from each subject were observed. RMSE calculations are applied to each proposed method. Subjective performance testing is also done by looking at the ECG wave graph. Table 1 shows the RMSE for each method used in this research.

Implementation of handling the incorrect data on ECG data using the EM method produces the smallest RMSE value for most attributes, the average RMSE obtained was 4.77. These results indicate that the EM method is the best method for handling missing data compared to regression. This can be caused by the EM algorithm being introduced to handle missing data with several data loss patterns and is a computationally simple method by offering analytical solutions in M-step [32]. The linear regression method produces the smallest RMSE value for ECG 4 and ECG 5 because the pattern incorrect in the data tends to be monotonous, as can be seen in Figure 2. 


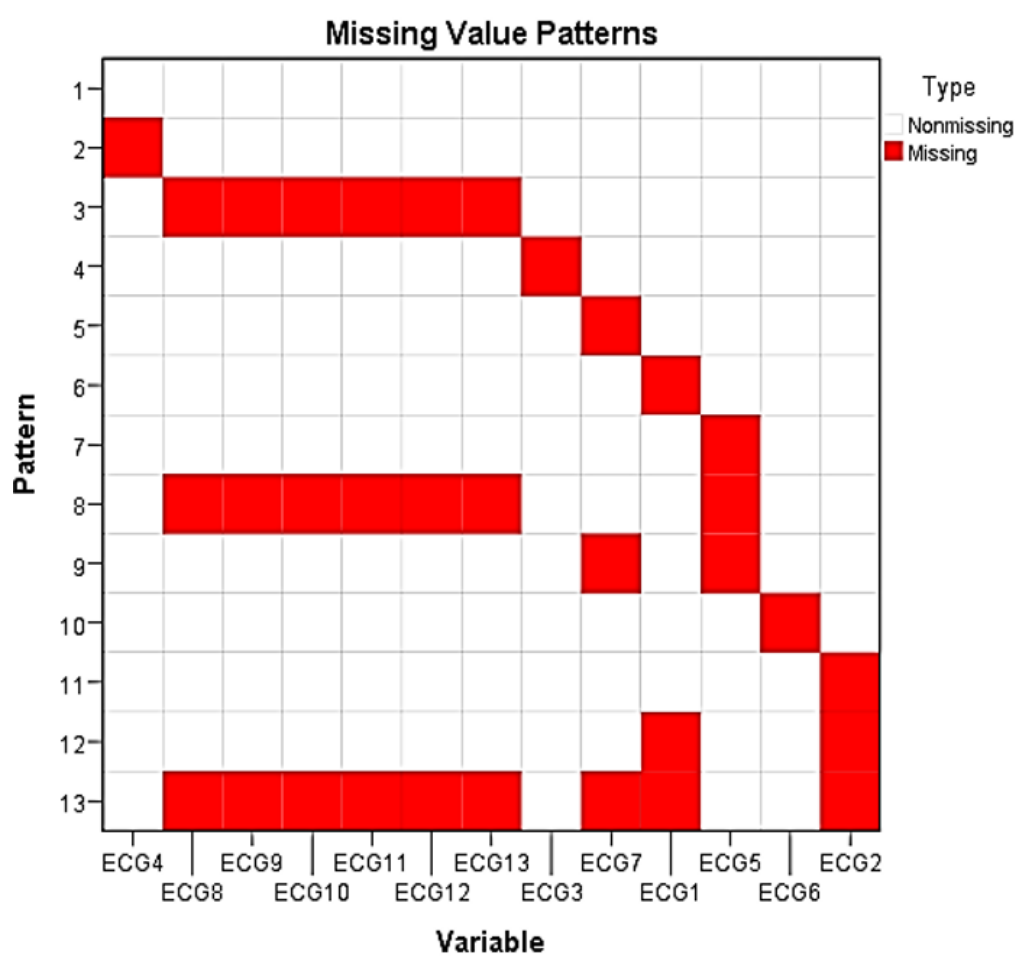

Figure 2. The Incorrect data pattern on ECG dataset

Table 1. RMSE for each method

\begin{tabular}{ccc}
\hline ECG & RMSE $($ EM) & RMSE $(\mathrm{RI})$ \\
\hline ECG1 & 6.25 & 6.28 \\
ECG2 & 3.57 & 5.75 \\
ECG3 & 5.74 & 10.03 \\
ECG4 & 3.82 & 3.78 \\
ECG5 & 8.36 & 6.73 \\
ECG6 & 3.58 & 5.26 \\
ECG7 & 3.88 & 7.50 \\
ECG8 & 1.51 & 2.27 \\
ECG9 & 4.76 & 6.40 \\
ECG10 & 6.66 & 10.73 \\
ECG11 & 6.95 & 8.02 \\
ECG12 & 5.49 & 11.02 \\
ECG13 & 1.50 & 2.42 \\
Mean & 4.77 & 6.63 \\
\hline
\end{tabular}

Figure 3(a) shows the ECG waves with incorrect data, Figure 3(b) shows the actual ECG waves, Figure 3(c) shows the predicted ECG waves using EM, and meanwhile, Figure 3(d) shows the predicted ECG waves using RI. As shown in Figure 3(a), the corrupted data occurs randomly on the observed signal line. This event occurs on all ECGs, especially when all nodes are active. Visually, the ECG waves that occur incorrect data are seen to have an impact on the appearance of waves such as spikes. This can result in reading errors by the application, especially for the heart rate estimation.

Figures 3(c) and 3(d) show an improvement in the ECG waveform through the two proposed method approaches. Visually, both have a similar waveform compared to the actual ECG wave. This happens because the value of the prediction error by the two methods is relatively small compared to the actual data. A comparison sample of prediction results to actual data that is stored in memory is shown in Table 2 . The proposed method in this study is able to correct the error sample point ECG signal by predicting it based on regression and probalistic approaches. The two proposed methods can be applied to resolve cases of incorrect data, typically in multi-node ECG monitoring systems that use ZigBee transmission. Based on the performance test results on the two methods, the EM method offers smaller error predictions compared to the RI method. However, the RI method has the advantage of simpler computation when applied to computers with low memory resources. 




(a)

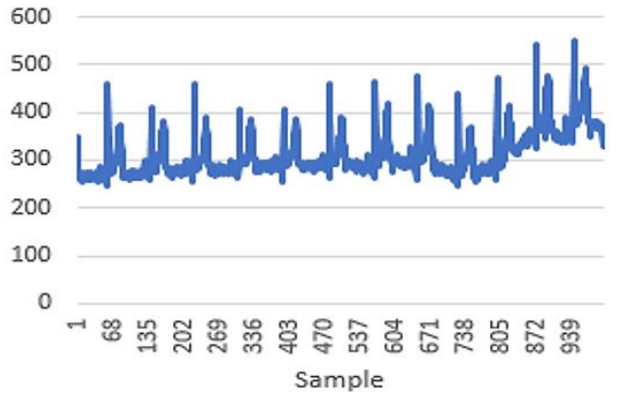

(c)

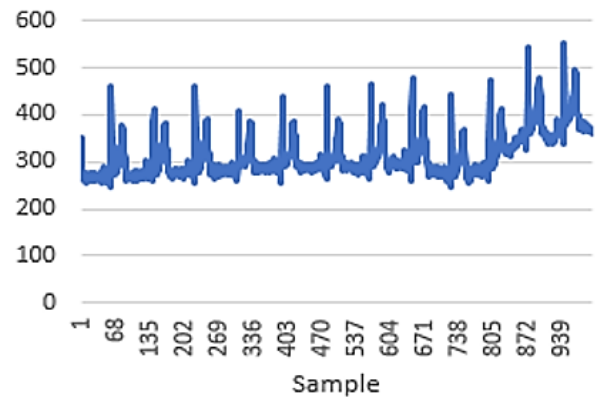

(b)

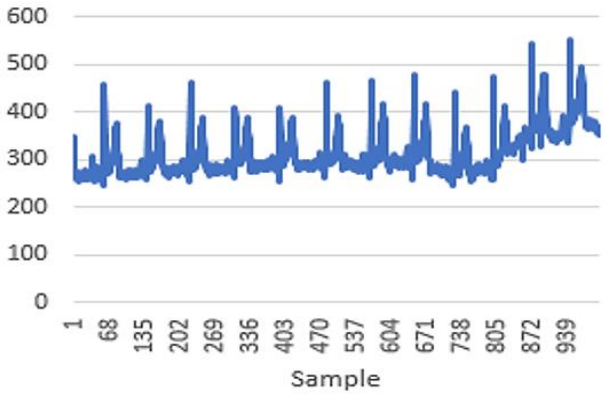

(d)

Figure 3. ECG waves (a) with incorrect data, (b) actual, (c) predicted-EM, and (d) predicted-Reg. imputation

Table 2. Comparison of actual and predicted data on 5 ECG waves

\begin{tabular}{cccc}
\hline \multirow{2}{*}{ Actual Data } & \multicolumn{2}{c}{ Data predicted } \\
& 260 & $259(0.3)$ & $269(3.4)$ \\
ECG-1 & 262 & $273(4.1)$ & $306(16.7)$ \\
& 274 & $284(3.6)$ & $285(4.01)$ \\
ECG-2 & 274 & $288(5.1)$ & $258(5.8)$ \\
& 266 & $285(7.1)$ & $296(11.2)$ \\
ECG-3 & 332 & $301(9.3)$ & $323(2.7)$ \\
& 273 & $307(12.4)$ & $363(32)$ \\
ECG-4 & 279 & $268(3.9)$ & $322(15.4)$ \\
& 296 & $292(1.3)$ & $393(32.7)$ \\
& 402 & $413(2.7)$ & $439(9.2)$ \\
ECG-5 & 398 & $405(1.7)$ & $404(1.5)$ \\
& 418 & $388(7.1)$ & $397(5.02)$ \\
& 322 & $338(4.9)$ & $337(4.6)$ \\
& 338 & $336(0.5)$ & $327(3.2)$ \\
& 315 & $336(6.67)$ & $327(3.8)$ \\
\hline
\end{tabular}

\section{CONCLUSION}

This study has succeeded in simulating predictions of the incorrect data in the case of multi-node ECG telemonitoring based on the ZigBee protocol. Two methods, namely regression imputation and EM are applied to overcome this problem. Performance measurement is done by calculating the RMSE between the ECG data predicted with the actual data. EM produces a smaller error compared to regression imputation. The simulation results show that the EM method has a lower predictive error value than the RI method with the average RMSE for the EM and RI methods are 4.77 and 6.63, respectively. However, on ECG 4 and ECG 5, the Regression Imputation performance has a smaller RMSE value. This phenomenon can be caused by the pattern of incorrect data that tends to be monotonous. Intuitively, the ECG wave from the two proposed approaches has a shape like the actual ECG wave. The proposed method in this study is expected to be applied to predict data in a multi-node monitoring system if incorrect data problems are encountered.

\section{REFERENCES}

[1] W. Liu, Q. Wu, J. Shen, J. Zhao, M. Zidan, and L. Tong, "An optimized quantum minimum searching algorithm with suresuccess probability and its experiment simulation with Cirq," Journal of Ambient Intelligence and Humanized Computing, vol. 12, no. 11, pp. 10425-10434, Nov. 2021, doi: 10.1007/s12652-020-02840-z. 
[2] M. Zidan, H. Eleuch, and M. Abdel-Aty, "Non-classical computing problems: Toward novel type of quantum computing problems," Results in Physics, vol. 21, Feb. 2021, Art. no. 103536, doi: 10.1016/j.rinp.2020.103536.

[3] M. Zidan, "A novel quantum computing model based on entanglement degree," Modern Physics Letters B, vol. 34, no. 35, Dec. 2020, Art. no. 2050401, doi: 10.1142/S0217984920504011.

[4] A.-H. Abdel-Aty, H. Kadry, M. Zidan, Y. Al-Sbou, E. A. Zanaty, and M. Abdel-Aty, "A quantum classification algorithm for classification incomplete patterns based on entanglement measure," Journal of Intelligent and Fuzzy Systems, vol. 38, no. 3, pp. 2809-2816, Mar. 2020, doi: 10.3233/JIFS-179566.

[5] M. Zidan et al., "Quantum classification algorithm based on competitive learning neural network and entanglement measure," Applied Sciences, vol. 9, no. 7, Mar. 2019, Art. no. 1277, doi: 10.3390/app9071277.

[6] M. Zidan, A.-H. Abdel-Aty, A. El-Sadek, E. A. Zanaty, and M. Abdel-Aty, "Low-cost autonomous perceptron neural network inspired by quantum computation," in AIP Conference Proceedings 1905, 2017, Art. no. 020005, doi: 10.1063/1.5012145.

[7] A. Sagheer, M. Zidan, and M. M. Abdelsamea, "A novel autonomous perceptron model for pattern classification applications," Entropy, vol. 21, no. 8, Aug. 2019, Art. no. 763, doi: 10.3390/e21080763.

[8] M. Zidan et al., "A quantum algorithm based on entanglement measure for classifying Boolean multivariate function into novel hidden classes," Results in Physics, vol. 15, Dec. 2019, Art. no. 102549, doi: 10.1016/j.rinp.2019.102549.

[9] J.-H. Lee, "Miniaturized human insertable cardiac monitoring system with wireless power transmission technique," Journal of Sensors, vol. 2016, pp. 1-7, 2016, doi: 10.1155/2016/5374574

[10] K. Padmavathi and K. S. Ramakrishna, "Detection of atrial fibrillation using autoregressive modeling," International Journal of Electrical and Computer Engineering (IJECE), vol. 5, no. 1, pp. 64-70, Feb. 2015, doi: 10.11591/ijece.v5i1.pp64-70.

[11] N. A. Nayan, R. Jaafar, and N. Sabrina Risman, "Development of respiratory rate estimation technique using electrocardiogram and photoplethysmogram for continuous health monitoring," Bulletin of Electrical Engineering and Informatics, vol. 7, no. 3, pp. 487-494, Sep. 2018, doi: 10.11591/eei.v7i3.1244

[12] S. Hadiyoso and S. Aulia, "Multipoint to point EKG monitoring berbasis ZigBee," Seminar Nasional Aplikasi Teknologi Informasi (SNATI), 2014.

[13] S. Hadiyoso, R. Mayasari, and A. Rizal, "The effect of mesh network on ECG data transmission by XBee toward the data error rate," ARPN Journal of Engineering and Applied Sciences, vol. 10, no. 20, pp. 9442-9446, 2015.

[14] H. Nugroho, N. P. Utama, and K. Surendro, "Class center-based firefly algorithm for handling missing data," Journal of Big Data, vol. 8, no. 1, Dec. 2021, Art. no. 37, doi: 10.1186/s40537-021-00424-y.

[15] T. R and D. A. Patil, "KNN and ARL based imputation to estimate missing values," Indonesian Journal of Electrical Engineering and Informatics (IJEEI), vol. 2, no. 3, Sep. 2014, doi: 10.11591/ijeei.v2i3.117.

[16] P. J. García-Laencina, J.-L. Sancho-Gómez, A. R. Figueiras-Vidal, and M. Verleysen, "K nearest neighbours with mutual information for simultaneous classification and missing data imputation," Neurocomputing, vol. 72, no. 7-9, pp. 1483-1493, Mar. 2009, doi: 10.1016/j.neucom.2008.11.026

[17] M. . Malarvizhi, "K-NN classifier performs better than K-Means clustering in missing value imputation," IOSR Journal of Computer Engineering, vol. 6, no. 5, pp. 12-15, 2012, doi: 10.9790/0661-0651215.

[18] M. Pampaka, G. Hutcheson, and J. Williams, "Handling missing data: Analysis of a challenging data set using multiple imputation," International Journal of Research and Method in Education, vol. 39, no. 1, pp. 19-37, Jan. 2016, doi: 10.1080/1743727X.2014.979146.

[19] C. G. Ng and M. S. B. Yusoff, "Missing values in data analysis: Ignore or impute?," Education in Medicine Journal, vol. 3, no. 1, Jun. 2011, doi: 10.5959/eimj.3.1.2011.or1.

[20] B. M. Marlin, Missing data problems in machine learning. University of Toronto, 2008

[21] Y. Dong and C.-Y. J. Peng, "Principled missing data methods for researchers," SpringerPlus, vol. 2, no. 1, Dec. 2013, Art. no. 222, doi: 10.1186/2193-1801-2-222.

[22] M. H. Gorelick, "Bias arising from missing data in predictive models," Journal of Clinical Epidemiology, vol. 59, no. 10, pp. 1115-1123, Oct. 2006, doi: 10.1016/j.jclinepi.2004.11.029.

[23] S. F. Morais, "Dealing with missing data: An application in the study of family history of hypertension," 2013.

[24] N. Demirel, The problem of missing data un regression analysis. Dokuz Eylül University, 2007.

[25] M. L. Yadav and B. Roychoudhury, "Handling missing values: A study of popular imputation packages in R," Knowledge-Based Systems, vol. 160, pp. 104-118, Nov. 2018, doi: 10.1016/j.knosys.2018.06.012.

[26] L. Beretta and A. Santaniello, "Nearest neighbor imputation algorithms: A critical evaluation," BMC Medical Informatics and Decision Making, vol. 16, no. S3, Jul. 2016, Art. no. 74, doi: 10.1186/s12911-016-0318-z.

[27] P. Hayati Rezvan, K. J. Lee, and J. A. Simpson, "The rise of multiple imputation: A review of the reporting and implementation of the method in medical research," BMC Medical Research Methodology, vol. 15, no. 1, Dec. 2015, Art. no. 30, doi: 10.1186/s12874-015-0022-1.

[28] C. Jittawiriyanukoon, "Evaluation of a multiple regression model for noisy and missing data," International Journal of Electrical and Computer Engineering (IJECE), vol. 8, no. 4, pp. 2220-2229, Aug. 2018, doi: 10.11591/ijece.v8i4.pp2220-2229.

[29] Y. Zhao and Q. Long, "Multiple imputation in the presence of high-dimensional data," Statistical Methods in Medical Research, vol. 25, no. 5, pp. 2021-2035, Oct. 2016, doi: 10.1177/0962280213511027.

[30] P. J. García-Laencina, J.-L. Sancho-Gómez, and A. R. Figueiras-Vidal, "Pattern classification with missing data: A review," Neural Computing and Applications, vol. 19, no. 2, pp. 263-282, Mar. 2010, doi: 10.1007/s00521-009-0295-6.

[31] W.-C. Lin and C.-F. Tsai, "Missing value imputation: A review and analysis of the literature (2006-2017)," Artificial Intelligence Review, vol. 53, no. 2, pp. 1487-1509, Feb. 2020, doi: 10.1007/s10462-019-09709-4.

[32] K. Zhang, R. Gonzalez, B. Huang, and G. Ji, "Expectation-maximization approach to fault diagnosis with missing data," IEEE Transactions on Industrial Electronics, vol. 62, no. 2, pp. 1231-1240, Feb. 2015, doi: 10.1109/TIE.2014.2336635.

[33] F. Qi, B. Huang, and E. C. Tamayo, "A Bayesian approach for control loop diagnosis with missing data," AIChE Journal, 2009, doi: 10.1002/aic.11964.

[34] E. Hartini, "Classification of missing values handling method during data mining: Review," Sigma Epsilon-Jurnal Ilmiah Teknologi Keselamatan Reaktor Nuklir, vol. 21, no. 2, pp. 49-60, 2017.

[35] E. D. Addo, "Performance comparison of imputation algorithms on missing at random data," East Tennessee State University, 2018

[36] H. Elçiçek, E. Akdoğan, and S. Karagöz, "The use of artificial neural network for prediction of dissolution kinetics," The Scientific World Journal, vol. 2014, pp. 1-9, 2014, doi: 10.1155/2014/194874.

[37] Y. Niu and S. Ye, "Data prediction based on support vector machine (SVM)-taking soil quality improvement test soil organic matter as an example," IOP Conference Series: Earth and Environmental Science, vol. 295, no. 2, Jul. 2019, Art. no. 012021, 
doi: 10.1088/1755-1315/295/2/012021.

[38] M. Singh, Implications of missing data designs with the use of a longitudinal dataset. University Muncie, 2017.

[39] Y. He, Missing data imputation for tree-based models. Los Angeles: University of California, 2006.

[40] I. Wasito and B. Mirkin, "Least squares algorithms with nearest neighbour techniques for imputing missing data values," $P h D$, School of Computer Science and Information Systems, Apr. 2003.

[41] R. J. A. Little and D. B. Rubin, Statistical analysis with missing data. Hoboken, NJ, USA: John Wiley \& Sons, Inc., 2002.

[42] N. J. Salkind, Encyclopedia of measurement and statistics. Thousand Oaks, Calif: SAGE Publications, 2006.

[43] J. L. Schafer and J. W. Graham, "Missing data: Our view of the state of the art," Psychological Methods, vol. 7, no. 2, pp. 147-177, 2002, doi: 10.1037/1082-989X.7.2.147.

[44] M. Watanabe and K. Yamaguchi, Eds., The EM Algorithm and Related Statistical Models. CRC Press, 2003.

[45] P. D. Allison, "Quantitative applications in the social sciences," in Missing Data, Thousand Oaks London New Delhi: Sage Publication, 2001

\section{BIOGRAPHIES OF AUTHORS}


Sugondo Hadiyoso (D) SC P received the Master in Electrical-Telecommunication Engineering from Telkom University, Bandung, Indonesia in March 2012. Since 2010, he joined as a Lecturer in the Diploma of Telecommunication Technology, School of Applied Science, Telkom University. He is also currently members of Biomedical Instrumentation Research Group in Telkom Univesity. His research interests are wireless sensor network, embedded system, logic design on FPGA and biomedical engineering. In 2018-present, he became a doctoral student in electrical engineering at the Bandung Institute of Technology. The focus of his doctoral research is signal processing and analysis of EEG waves. Sugondo also actively writes articles in international conferences and journals in the field of biomedical instrumentation and biomedical signal processing including biometric systems based on ECG or EEG signals. He can be contacted at email: sugondo@telkomuniversity.ac.id.

Heru Nugroho (D) 8. SC P is a lecturer in School of Applied Science, Telkom University with field of Interest in Information System; IT Governance; Data Analytics; and Machine Learning. Graduated Master of Engineering from School of Electrical Engineering and Informatics, Bandung Institute of Technology. He can be contacted at email: heru@tass.telkomuniversity.ac.id.

Tati Latifah Erawati Rajab (iD SOS SC is a Professor in School of Electrical Engineering and Informatics, Bandung Institute of Technology. Getting a Doctoral degree from Electrotechnique, Institute National Polytechnique de Grenoble-France. Research interest in Image processing and Recognition, Medical Imaging, e-Health. She can be contacted at email: tati@stei.itb.ac.id.

Surendro Kridanto (D) SC PC is a lecturer in School of Electrical Engineering and Informatics, Bandung Institute of Technology. Getting a $\mathrm{PhD}$ degree in Computer Science from Keio University-Japan. Research interest in software engineering, information system, and information science. He can be contacted at email: endro@informatika.org. 